\title{
Inhibition of methionine adenosyltransferase II induces FasL expression, Fas-DISC formation and caspase-8-dependent apoptotic death in T leukemic cells
}

Tanvi S Jani, ${ }^{1,}$, Leila Gobejishvilii, ${ }^{2,}$, Prachi T Hote ${ }^{1}$, Aditya S Barve ${ }^{2}$, Swati Joshi-Barve ${ }^{2}$, Giorgi Kharebava ${ }^{3}$, Jill Suttles ${ }^{4}$, Theresa Chen ${ }^{1}$, Craig J McClain ${ }^{1,2,5}$, Shirish Barve ${ }^{1,2}$

${ }^{I}$ Department of Pharmacology and Toxicology, University of Louisville Medical Center, Louisville, KY 40292, USA; ${ }^{2}$ Department of Internal Medicine, University of Louisville Medical Center, Louisville, KY 40292, USA; ${ }^{3}$ Department of Neurological Surgery, KSCIRC, Louisville, KY 40292, USA; ${ }^{4}$ Department of Microbiology and Immunology, Louisville, KY 40292, USA; ${ }^{5}$ Louisville VA Medical Center, Louisville, KY 40292, USA

Methionine adenosyltransferase II (MAT II) is a key enzyme in cellular metabolism and catalyzes the formation of S-adenosylmethionine (SAMe) from L-methionine and ATP. Normal resting T lymphocytes have minimal MAT II activity, whereas activated proliferating $T$ lymphocytes and transformed $T$ leukemic cells show significantly enhanced MAT II activity. This work was carried out to examine the role of MAT II activity and SAMe biosynthesis in the survival of leukemic T cells. Inhibition of MAT II and the resultant decrease in SAMe levels enhanced expression of FasL mRNA and protein, and induced DISC (Death Inducing Signaling Complex) formation with FADD (Fasassociated Death Domain) and procaspase-8 recruitment, as well as concomitant increase in caspase-8 activation and decrease in c-FLIPs levels. Fas-initiated signaling induced by MAT II inhibition was observed to link to the mitochondrial pathway via Bid cleavage and to ultimately lead to increased caspase-3 activation and DNA fragmentation in these cells. Furthermore, blocking MAT $2 A$ mRNA expression, which encodes the catalytic subunits of MAT II, using a small-interfering RNA approach enhanced FasL expression and cell death, validating the essential nature of MAT II activity in the survival of $\mathrm{T}$ leukemic cells.

Keywords: methionine adenosyltransferase, S-adenosylmethionine, FasL, FADD, caspase-8

Cell Research (2009) 19:358-369. doi: 10.1038/cr.2008.314; published online 2 December 2008

\section{Introduction}

Methionine adenosyltransferase (MAT) is a key enzyme in cellular metabolism. It is the only enzyme that catalyzes the transfer of the adenosyl moiety from ATP to L-methionine, giving rise to $S$-adenosylmethionine (Adomet, SAM, hereafter referred to as SAMe) [1]. SAMe plays a critical role in cellular metabolism by

*These authors contributed equally to this work

Correspondence: Shirish Barve

Department of Medicine, Pharmacology and Toxicology, University of Louisville Medical Center, 511 S. Floyd, MDR Bldg, Rm\#526, Louisville, KY 40202, USA;

Tel: +1-502-852-5245; Fax: +1-502-852-8927

E-mail: shirish.barve@louisville.edu

Received 30 January 2008; revised 30 Juny 2008; accepted 31 July 2008; published online 2 December 2008 functioning as the major methyl donor via methyltransferases to various biomolecules that are essential for normal functioning of the cell, such as DNA, RNA, phospholipids, lipids, proteins, etc. [2, 3]. Apart from being the principal methyl donor, SAMe also acts as the sole source of the propylamine moiety required for polyamine synthesis, which is vital for cellular growth and differentiation, and also serves as an obligatory intermediate in the transsulfuration pathway by producing homocysteine (Hcy), which is required for glutathione (GSH) synthesis $[2,3]$.

In mammals, MAT is encoded by different genes, MAT 1 and MAT 2A/2B [4]. MAT 1 encodes for the $\alpha 1$ catalytic subunit, which either forms a tetramer, leading to the synthesis of the MAT I isozyme, or forms a dimer, leading to the formation of the MAT III isozyme [4]. Products of MAT $2 A$ (catalytically active subunits $\alpha 2 / \alpha 2^{\prime}$ ) 
and $M A T 2 B$ (catalytically inactive regulatory subunit $\beta$ ) form a hetero-oligomer $\left(\alpha 2 \alpha 2^{\prime} \beta\right)$ to generate the MAT II isozyme [4-8]. MAT is found in almost all cells; the MAT isozymes are highly conserved throughout all species and vary mainly in their structure, kinetic properties and tissue distribution $[4,8]$. MAT I and MAT III are mainly expressed in the liver and are referred to as the hepatic MAT isozymes. MAT II, on the other hand, is the non-hepatic MAT and has a broader tissue distribution that includes the brain, kidney, lens, erythrocytes, fetal liver and human lymphocytes $[4,8]$. In relation to the non-hepatic MAT II isozyme, it has been shown that in resting lymphocytes, the MAT $2 A$ gene initially encodes for a less active precursor subunit $\lambda$, which is further processed to the catalytically active $\alpha 2 / \alpha 2^{\prime}$ subunits upon $\mathrm{T}$ cell activation and proliferation [9]. Accordingly, MAT II activity is constitutively expressed in stimulated, actively dividing T cells as well as T leukemic cells [9-11].

This work was aimed at examining the role of MAT II and SAMe biosynthesis in the survival of T leukemic cells. MAT II activity was inhibited using cycloleucine (1-aminocyclopentane-1-carboxylic acid), a cyclic analogue of methionine, which acts as a specific inhibitor of MAT [12, 13]. Our results show that inhibition of MAT II by cycloleucine and subsequent loss of intracellular SAMe pools induces Fas-mediated apoptotic cell death in leukemic T cells. MAT II inhibition results in the induction of FasL gene expression, a decrease in c-FLIP levels and a corresponding increase in caspase- 8 activity, leading to Fas-mediated apoptotic cell death. Our data indicate that MAT II and SAMe biosynthesis play a key role in the survival of leukemic $\mathrm{T}$ cells and identifies this pathway as a novel target for cancer therapy of $\mathrm{T}$ lymphocytic leukemia.

\section{Results}

Jurkat and MOLT-4 T leukemic cell lines were used to examine the effect of MAT II inhibition on the survival of $\mathrm{T}$ leukemic cells. Cycloleucine is an amino acid analogue of methionine and it functions as a selective inhibitor of MAT.

Effect of cycloleucine on MAT II activity and intracellular SAMe levels

To determine the effect of MAT II inhibition, Jurkat $\mathrm{T}$ leukemic cells were treated with increasing concentrations of the selective MAT inhibitor, cycloleucine (10, 20, 40, 60 and $80 \mathrm{mM}$ ), for a period of $18 \mathrm{~h}$. Cellular MAT II activity was measured by monitoring the amount of SAMe formed by HPLC. As expected, cycloleucine blocked MAT II activity in a dose-dependent manner,
A

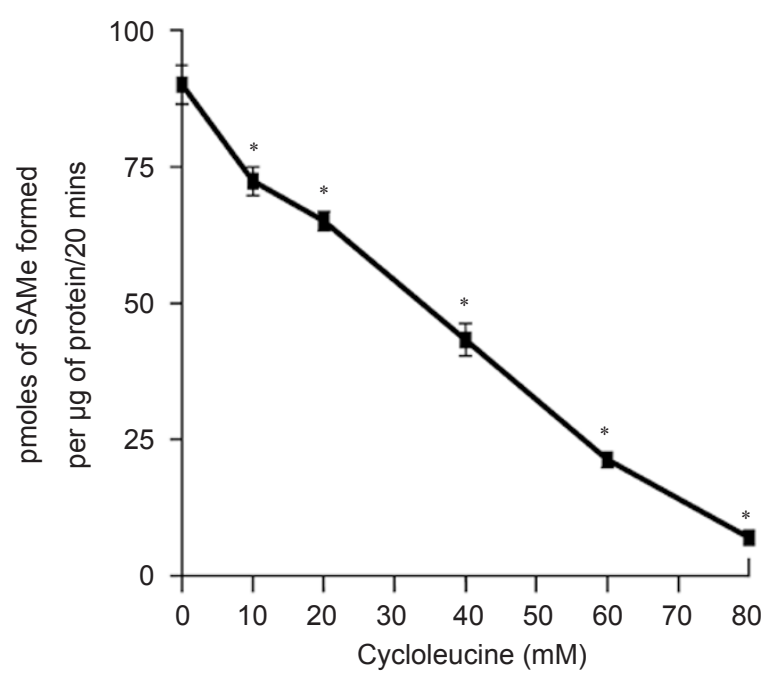

B

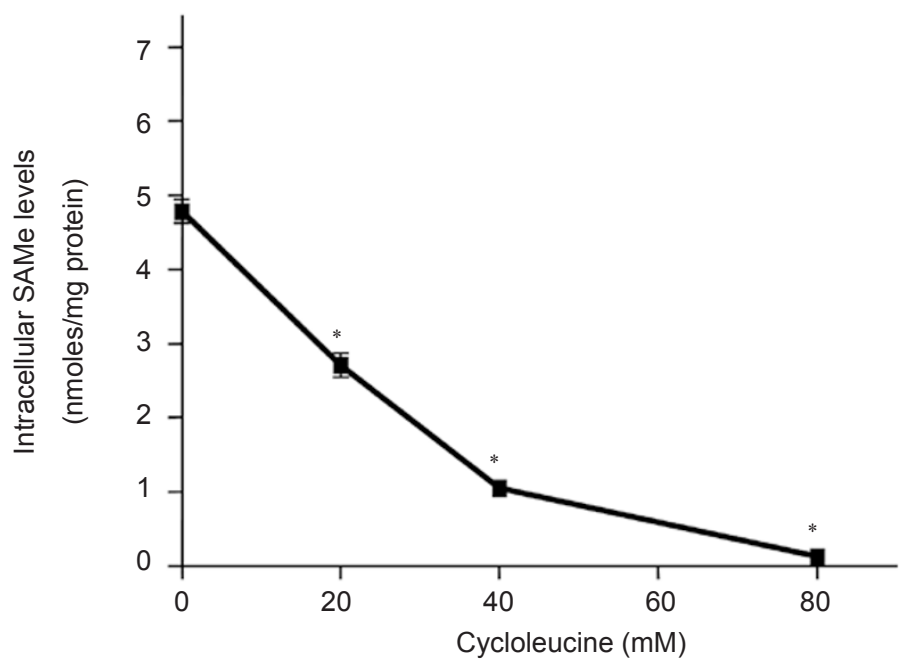

Figure 1 Cycloleucine inhibits MAT II activity and decreases intracellular SAMe levels. (A) Jurkat cells were either left untreated (UT) or treated with increasing concentrations of cycloleucine $(10-80 \mathrm{mM})$ for $18 \mathrm{~h}$. Cell extracts were prepared and MAT II activity was measured as described in Materials and Methods. Data are expressed as pmol of SAMe formed per $\mu \mathrm{g}$ of protein (extract) in $20 \mathrm{~min}$. Results from three experiments are shown as mean \pm S.E.M. $* P<0.001$ compared with UT. (B) Jurkat cells were either left untreated (UT) or treated with increasing concentrations of cycloleucine for $18 \mathrm{~h}$. Intracellular SAMe concentration was determined by HPLC as described in Materials and methods. Data are expressed as nmol of SAMe per mg of protein. Results from three experiments are shown as mean \pm S.E.M. $* P<0.001$ compared with UT. 
A

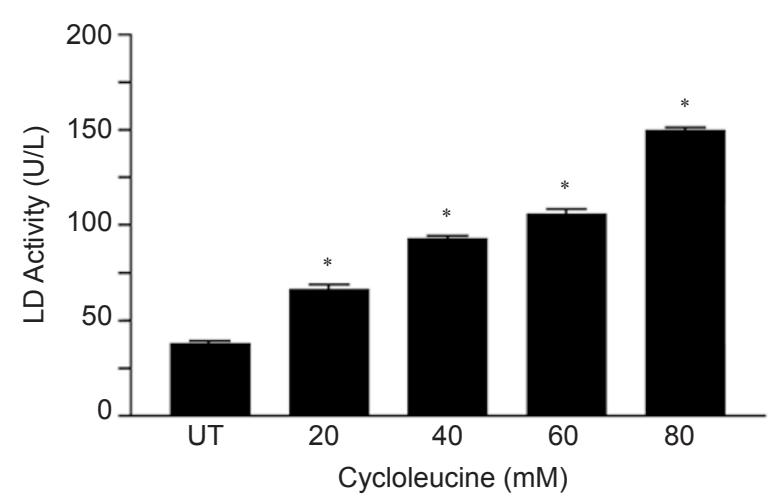

B

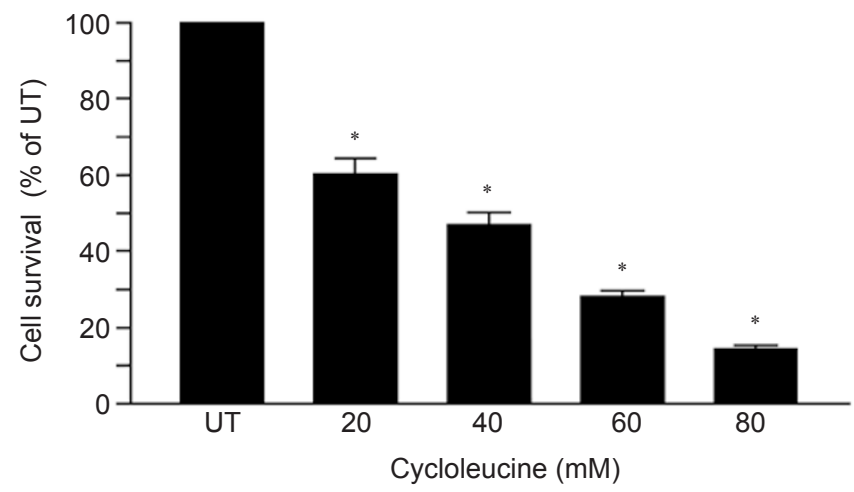

C

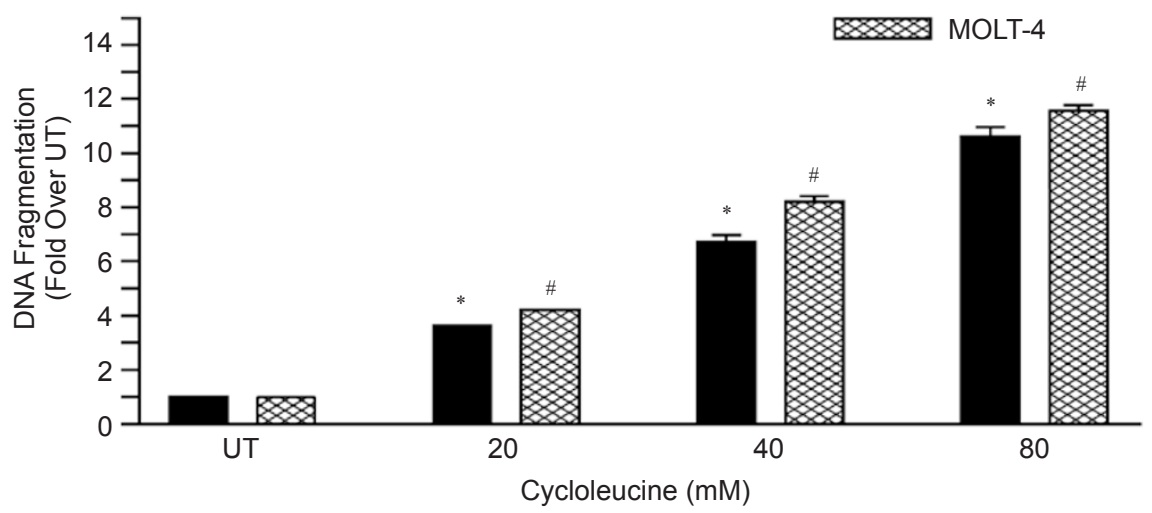

Figure 2 Inhibition of MAT II activity leads to decreased cell survival and increased DNA fragmentation. Jurkat T cells were either left untreated (UT) or treated with $20-80 \mathrm{mM}$ of cycloleucine for $18 \mathrm{~h}$. (A) After $18 \mathrm{~h}$, cell survival was measured by lactate dehydrogenase assay. Data are expressed as LD activity (fold over UT). Results from three separate experiments are shown as mean \pm S.E.M. $* P<0.001$ compared with UT. (B) After $18 \mathrm{~h}$, cell survival was measured by Trypan Blue exclusion assay. Data are expressed as \% decrease in cell survival as compared with untreated cells. Results from three separate experiments are shown as mean \pm S.E.M. ${ }^{*} P<0.001$ compared with UT. (C) DNA fragmentation induced by MAT II inhibition in Jurkat and MOLT-4 T leukemic cells was quantified by DNA fragmentation ELISA at $405 \mathrm{~nm}$. Data from three separate experiments, run in duplicate, are expressed as fold over UT \pm S.E.M. $* P<0.001$ compared with UT in Jurkat T leukemic cells, ${ }^{\#} P<0.001$ compared with UT in MOLT-4 T leukemic cells.

with $\sim 50 \%$ inhibition achieved at a concentration of 40 $\mathrm{mM}$ (Figure 1A).

As MAT is the critical enzyme that catalyzes the conversion of methionine into SAMe, the effect of inhibition of MAT II activity on intracellular SAMe levels was further evaluated. Intracellular concentrations of SAMe were measured by HPLC in deproteinized cell extracts prepared from cycloleucine-treated cells. Treatment of cells with cycloleucine resulted in a dose-dependent decrease in intracellular SAMe levels (Figure 1B), which corresponded with the inhibition of MAT II activity seen in Figure 1A. An approximate decrease of $50 \%$ in intracellular SAMe levels was seen at $20 \mathrm{mM}$ cycloleucine.
Effect of MAT II inhibition on cell viability and apoptotic cell death

Since MAT II is critical for T cell proliferation and activation, the effect of MAT II inhibition on viability of Jurkat $\mathrm{T}$ leukemic cells was examined by the lactate dehydrogenase (LDH) assay and by Trypan Blue dye exclusion. MAT II inhibition by cycloleucine led to a dosedependent increase in cell death as determined by the LDH assay (Figure 2A) and a decrease in cell viability as documented by the Trypan Blue dye exclusion assay (Figure 2B) after 18-24 h as compared to untreated cells. A $>50 \%$ loss of survival was observed at a concentration of $40 \mathrm{mM}$ cycloleucine. 
To determine whether the observed cell death caused by inhibition of MAT II activity occurred by induction of apoptosis, DNA fragmentation was evaluated quantitatively by cell death ELISA in MAT II-inhibited cells. Cycloleucine induced a dose-dependent increase in apoptotic cell death as a result of MAT II inhibition (Figure 2C). Similar to Jurkat T leukemic cells, inhibition of MAT II activity by cycloleucine also induced apoptotic cell death in a different $\mathrm{T}$ leukemic cell line (MOLT-4) (Figure 2C). These data strongly indicate that inhibition of MAT II activity may decrease the survival of T leukemic cells by inducing apoptotic cell death.

\section{Effect of MAT II inhibition on FasL expression}

As FasL is known to play a major role in the induction of apoptosis in T lymphocytes [14], we examined the effect of MAT II inhibition on FasL expression. FasL protein expression was analyzed by immunoblotting. As shown in Figure 3A, inhibition of MAT II activity led to a dose-dependent increase in FasL protein levels. Importantly, the level of FasL expression induced in re-

A

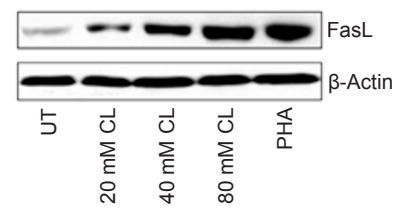

C

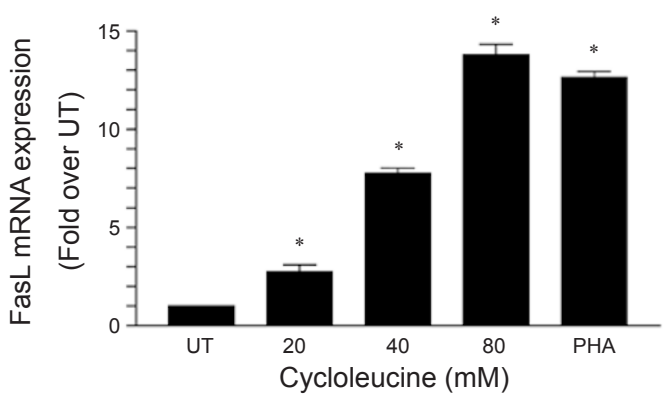

Figure 3 MAT II inhibition augments FasL expression. Total RNA and total cellular extracts were obtained from Jurkat $T$ leukemic cells after treatment with cycloleucine for $6 \mathrm{~h}$. Cells treated with PHA for $6 \mathrm{~h}$ served as a positive control. (A) FasL expression was assessed by immunoblot analysis and the data are representative of three separate experiments. (B) FasL mRNA levels were determined by RT-PCR and visualized by $10 \%$ polyacrylamide gel electrophoresis. The data are representative of the results obtained in three separate experiments. (C) FasL mRNA levels were quantified by real-time PCR. Data from three separate experiments, run in duplicate, are expressed as fold over UT \pm S.E.M. $* P<0.001$ compared with UT values.

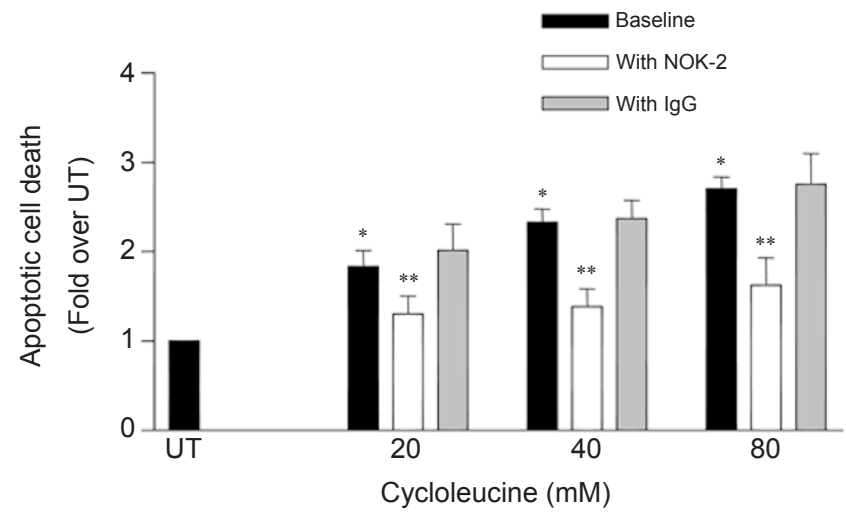

Figure 4 FasL neutralizing antibody, NOK-2, attenuates apoptosis in MAT II inhibited cells. Jurkat T cells were treated with 50 $\mu \mathrm{g} / \mathrm{ml}$ of NOK-2 antibody or isotype control IgG after $1 \mathrm{~h}$ treatment with cycloleucine (20-80 mM). Apoptotic death was measured by DNA fragmentation ELISA. Data from three separate experiments were normalized to UT (which was set to 1) and are expressed as mean \pm S.E.M. $* P<0.05$ compared with UT values, $* * P<0.05$ compared with the corresponding baseline values.

sponse to decreased MAT II activity was observed to be comparable to that induced by the T cell mitogen, phytohemaglutinin (PHA), which is known to induce FasL and cause activation-induced apoptotic death in T cells.

Further, to determine whether the up-regulation of FasL in response to MAT II inhibition was at the transcription level, we analyzed FasL mRNA expression by real-time PCR as well as reverse transcriptase polymerase chain reaction (RT-PCR). The data showed that in correlation with the up-regulation of FasL protein, FasL mRNA expression increased in a dose-dependent manner upon treatment with cycloleucine (Figure 3B and 3C).

In order to test the functional relevance of FasL expression in MAT II inhibition-induced cell death, we examined whether blocking Fas/FasL interaction would protect Jurkat $\mathrm{T}$ leukemic cells. We used the anti-FasL IgG antibody (NOK-2), which recognizes and neutralizes both membrane-bound as well as soluble forms of human FasL and thus interferes with Fas/FasL interactions and inhibits Fas signaling. Cells were incubated with NOK-2 antibody after $1 \mathrm{~h}$ of cycloleucine treatment and apoptotic cell death was monitored by quantifying DNA fragmentation. NOK-2 antibody significantly attenuated DNA fragmentation (Figure 4), whereas treatment with the $\mathrm{IgG}_{2 \mathrm{a}}$ isotype specific antibody (IgG) (negative control) did not protect the cells from apoptosis (Figure 4). These data indicate a direct and specific association between MAT II inhibition and FasL-dependent apoptotic cell death. 
A

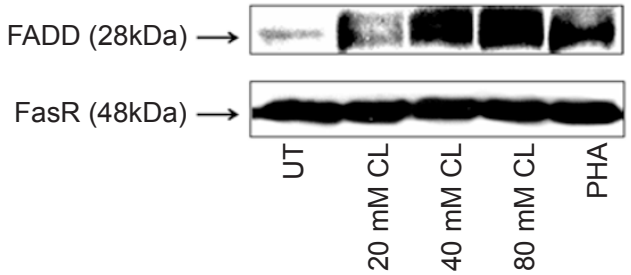

C

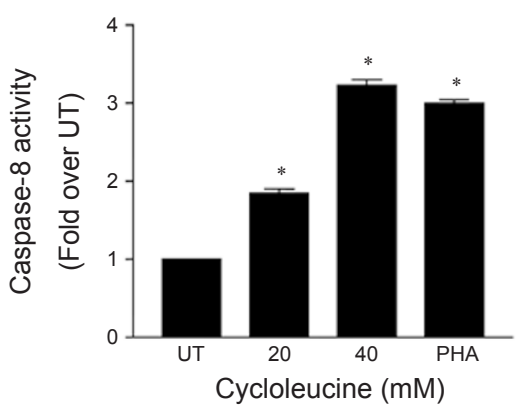

B

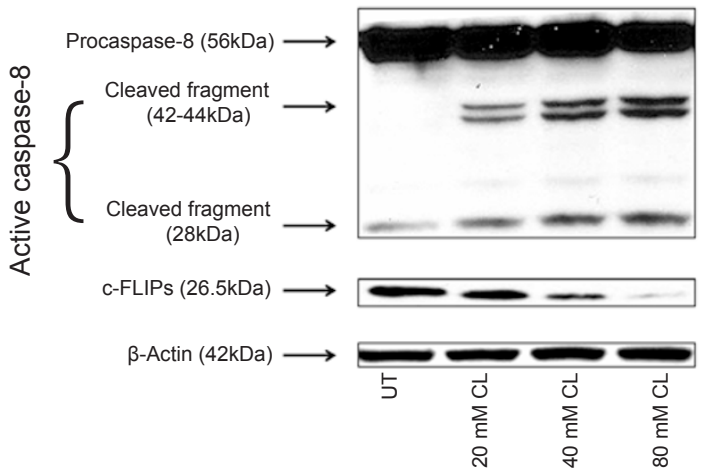

D

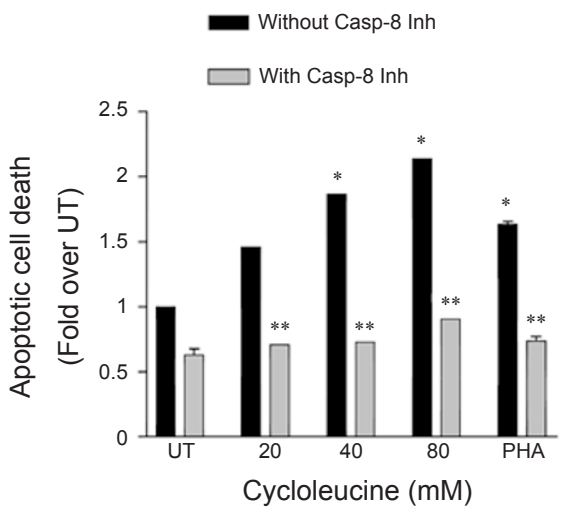

E

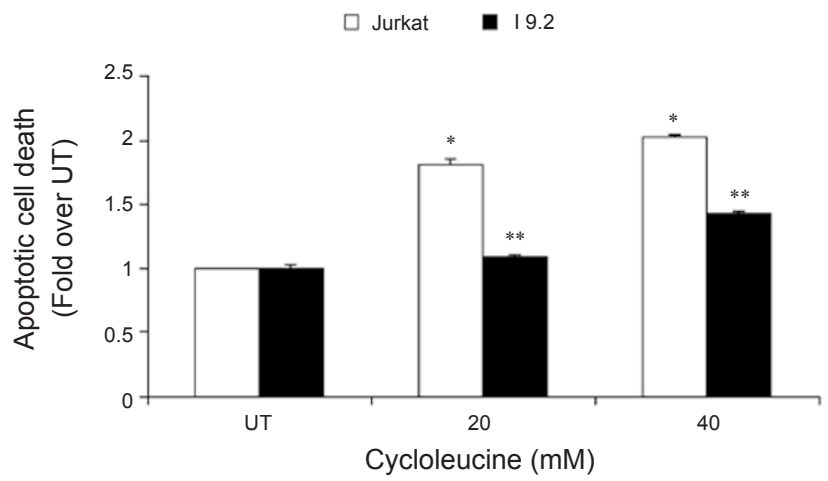

Figure 5 Inhibition of MAT II activity enhances FADD recruitment to the FasR, down-regulates CFLIPs levels and augments caspase-8 activation leading to caspase-8-dependent apoptosis. Cellular lysates were obtained from Jurkat T leukemic cells treated with cycloleucine $(\mathrm{CL}, 20-80 \mathrm{mM})$ for $6 \mathrm{~h}$. Cells treated with PHA served as a positive control. (A) Immunoprecipitation with Fas receptor antibody and subsequent immunoblot analysis with FADD were performed to evaluate the levels of FADD recruited at the FasR in the MAT II-inhibited leukemic T cells. FasR levels were also determined for loading control. The data depicted are representative of results found in three separate experiments. (B) Immunoblot analysis was performed for caspase- 8 and c-FLIPs. Blots were stripped and reprobed with antibody to $\beta$-actin to ensure equivalent loading. The data are representative of three separate experiments. (C) Caspase-8 activity was measured using the caspase-8 colorimetric activity assay kit (Chemicon International, Temecula, CA, USA). Results from three separate experiments are shown as mean \pm S.E.M. $* P<0.001$ compared with UT values. (D) Jurkat $T$ cells were pretreated with caspase-8 inhibitor $(100 \mu \mathrm{M})$ for $1 \mathrm{~h}$ prior to treatment with cycloleucine. Apoptotic cell death was measured by DNA fragmentation ELISA assay. Data from three separate experiments, run in duplicate, are expressed as fold over UT and shown as \pm S.E.M. $* P<0.001$ compared with UT values. ${ }^{*} P<0.001$ compared with baseline values. (E) Caspase-8 mutant Jurkat T cells (I 9.2) and wild-type Jurkat T cells were treated with cycloleucine (20 and $40 \mathrm{mM})$ for $6 \mathrm{~h}$. Apoptotic cell death was measured by DNA fragmentation ELISA assay. Data from three separate experiments, run in duplicate, are expressed as fold over UT and shown as \pm S.E.M. ${ }^{*} P<0.05$ compared with UT values. ${ }^{* *} P<0.02$ compared with wild-type Jurkat. 
Effect of MAT II inhibition on FADD(Fas-associated Death Domain) recruitment, caspase- 8 activation and cFLIPS expression

To further elucidate the effect of MAT II inhibition, components of the Fas/FasL apoptotic pathway were examined in MAT II-inhibited Jurkat T leukemic cells. Ligation of FasR (Fas receptor) with FasL induces the formation of DISC (Death Inducing Signaling Complex), which involves the recruitment of a pro-apoptotic adapter protein, FADD to the FasR [15-17]. This binding further signals the recruitment and subsequent activation of caspase-8 (FLICE). Initially, the recruitment of FADD to FasR was examined by using a Fas antibody to immunoprecipitate the DISC from the lysates (normalized for proteins) of MAT II-inhibited cells. The immunoprecipitates were then resolved on a $10 \%$ SDS gel and examined by immunoblot analysis using antibodies against FADD and FasR. An increase in FADD recruitment was observed in the MAT II-inhibited Jurkat T leukemic cells as compared with the control cells. The amount of FADD recruitment was greater than or equal to the amount of FADD recruitment seen in cells treated with PHA $(5 \mu \mathrm{g} / \mathrm{ml})$, a known positive control (Figure 5A). Our data indicate that MAT II inhibition leads to increased FADD recruitment to the FasR without affecting FasR expression.

FADD mediates the recruitment and activation of the initiator caspase, caspase-8. Hence, the effect of MAT II inhibition on procaspase- 8 activation was examined. MAT II inhibition led to an increase in the activation/ cleavage of procaspase- $8(56 \mathrm{kDa})$ and generation of cleaved fragments (p42-44 and p28 kDa) (Figure 5B). The enhancement in the cleavage of procaspase- 8 seen by the immunoblot analysis was also reflected by an increase in caspase- 8 enzymatic activity in Jurkat $T$ cells treated with cycloleucine for $8 \mathrm{~h}$ as compared with the untreated cells (Figure 5C). Importantly, a caspase-8-specific inhibitor abrogated the apoptotic cell death induced by MAT II inhibition in these cells (Figure 5D). To further validate the role of caspase- 8 in apoptosis caused by MAT II inhibition, caspase-8 mutant Jurkat T cells (I 9.2) were treated with cycloleucine and DNA fragmentation was quantified to assess apoptosis. Caspase-8-deficient I 9.2 cells were significantly resistant to apoptosis compared with wild-type Jurkat T cells (Figure 5E).

It is known that both long and short variants of cFLIP (FLICE-inhibitory protein) are inhibitors of death
A

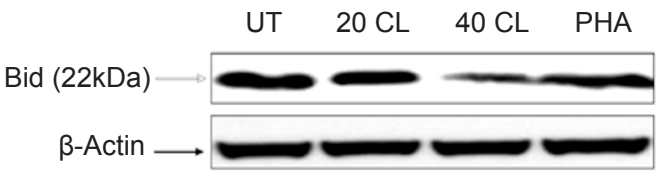

B

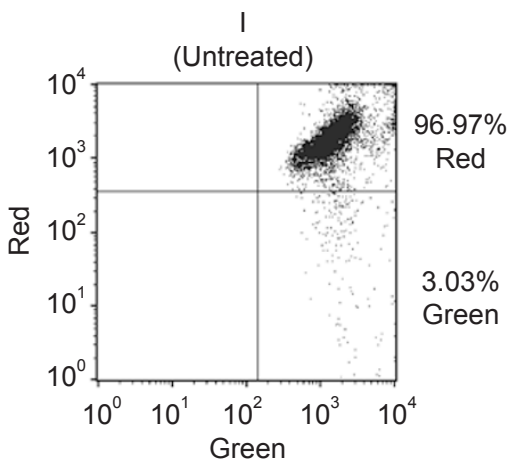

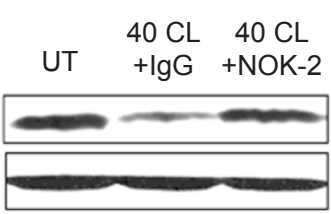

II

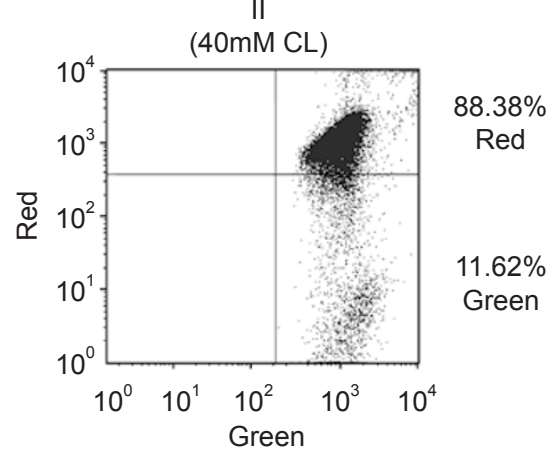

Figure 6 Inhibition of MAT II activity leads to increased Bid truncation and disruption of mitochondrial transmembrane potential in Jurkat T leukemic cells. (A) Immunoblot analysis for Bid was performed on cellular extracts obtained from Jurkat $T$ leukemic and I 9.2 cells treated for $8 \mathrm{~h}$ with cycloleucine (CL). Jurkat T cells were treated with $50 \mu \mathrm{g} / \mathrm{ml}$ of NOK-2 antibody or isotype control lgG after $1 \mathrm{~h}$ treatment with cycloleucine $40 \mathrm{mM}$. Cells stimulated with PHA served as a positive control. Blots were stripped and reprobed with antibody to $\beta$-actin to ensure equivalent loading. The data are representative of three separate experiments. (B) Changes in mitochondrial membrane potential induced by MAT II inhibition were analyzed in Jurkat T leukemic cells treated with cycloleucine $(40 \mathrm{mM} ; 12 \mathrm{~h})$ using JC-1 dye $(5 \mu \mathrm{g} / \mathrm{ml})$ as described in Materials and methods. The lower right quadrant represents cells that have undergone mitochondrial permeability transition (MPT) leading to an increase in the percentage of MAT II-inhibited cells (II), showing green florescence as compared with untreated cells (I). 
receptor-mediated apoptosis $[18,19]$. As, in lymphocytes, the c-FLIPS isoform is anti-apoptotic and inhibits Fas-mediated apoptosis by acting as a dominant-negative inhibitor of caspase-8 [20-23], we examined the levels of c-FLIP $_{\mathrm{S}}$ in MAT II-inhibited T cells. Cycloleucine treatment led to a substantial decrease in c-FLIPs levels in a dose-dependent manner (Figure 5B).

These data strongly suggest that inhibition of MAT II activity leads to the up-regulation of FasL expression followed by a down-regulation of c-FLIP levels and concomitant activation of caspase- 8 , leading to caspase8-dependent apoptotic cell death in T leukemic cells.

Effect of MAT II inhibition on Bid truncation, mitochondrial transmembrane potential $\left(\Delta \Psi_{m}\right)$ and caspase-3 maturation

In type II cells, like the Jurkat T leukemic cells, Fasmediated caspase- 8 activation can initiate the mitochondrial apoptotic pathway by cleaving Bid, a pro-apoptotic member of the Bcl-2 family. Upon activation, the truncated Bid (t-Bid) translocates to the mitochondrial membrane and causes a decrease in the mitochondrial transmembrane potential $\left(\Delta \Psi_{\mathrm{m}}\right)$, initiating mitochondrial apoptotic signaling and leading ultimately to the activation of the effector caspases (e.g. caspase-3) [23, 24]. Hence, to evaluate the involvement of the mitochondrial pathway in Fas-mediated apoptotic death in MAT IIinhibited cells, Bid cleavage, decrease in $\Delta \Psi_{\mathrm{m}}$ and maturation of caspase-3 were examined.

In correspondence with caspase- 8 activation, MAT II inhibition induced significant Bid cleavage as compared with the untreated cells, which was prevented by NOK-2 treatment (Figure 6A); in addition, caspase- 8 mutant $\mathrm{T}$ cells (I 9.2) were resistant to Bid cleavage upon cycloleucine treatment (Figure 6A). Further, MAT II inhibition of Jurkat $\mathrm{T}$ cells resulted in a dose-dependent dissipation of mitochondrial $\Delta \Psi_{\mathrm{m}}$ as indicated by an increase in the percentage of cells showing green fluorescence from $3.03 \%$ in untreated cells to $11.62 \%$ in cells treated with $40 \mathrm{mM}$ cycloleucine (Figure 6B). The effect of the above events on the activation of caspase-3 was further determined.

Immunoblot analysis showed that the $32-\mathrm{kDa}$ procaspase-3 was cleaved in cycloleucine-treated cells and the amount of cleaved caspase-3 fragments correlated with MAT II inhibition (Figure 7A). Caspase-3 activity in MAT II-inhibited leukemic T cells was also quantified by fluorimetric analysis using a specific Ac-DEVD-AMC substrate. In agreement with the immunoblot analysis, caspase-3 activity was observed to increase substantially in Jurkat T leukemic cells undergoing apoptosis due to MAT II inhibition induced by cycloleucine (Figure 7B). Similar induction of caspase- 3 activity caused by MAT II
A

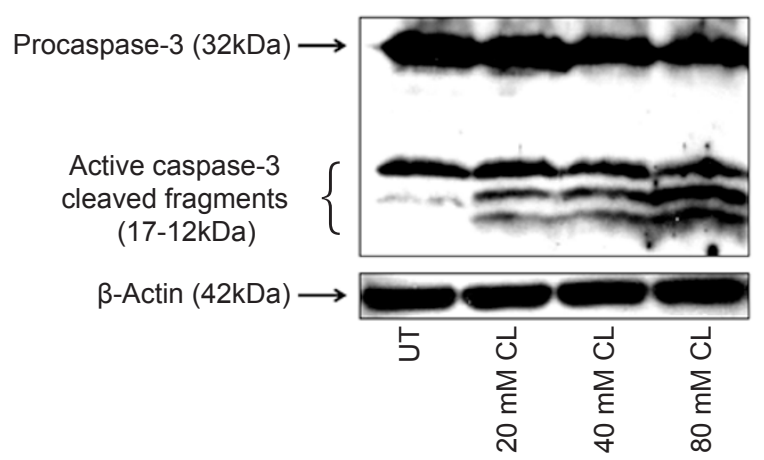

\section{B}

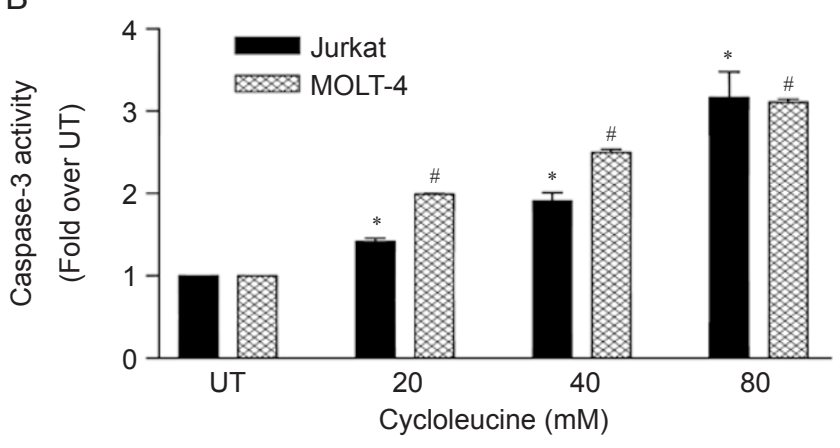

C

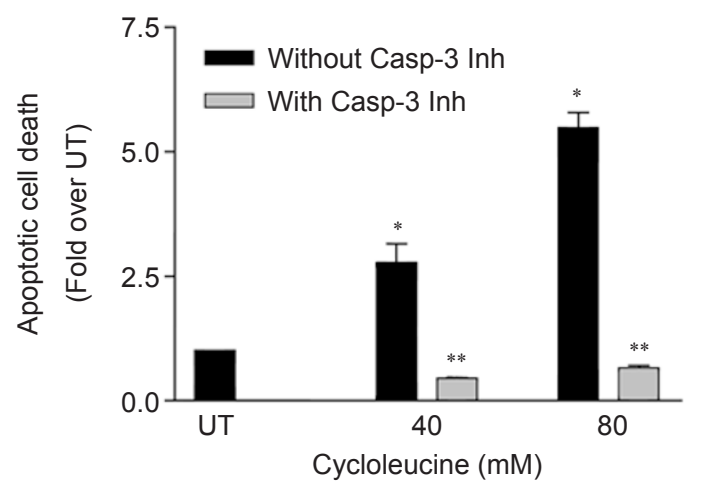

Figure 7 MAT II inhibition results in increased caspase-3 activation in Jurkat and MOLT-4 T leukemic cells. Leukemic T cells were treated with cycloleucine (20-80 mM). (A) Procaspase-3 and caspase- 3 levels were detected in Jurkat $T$ cells treated for $18 \mathrm{~h}$ using the immunoblotting technique. The gel picture is representative of the results obtained in three separate experiments. (B) Caspase-3 activity was measured in cell lysates obtained from Jurkat and MOLT-4 T leukemic cells that were treated for $6 \mathrm{~h}$. The data are expressed as fold over UT. Results from three separate experiments run in duplicates are shown as mean \pm S.E.M. ${ }^{*} P<0.001$ compared with UT in Jurkat T leukemic cells, ${ }^{\#} P<0.001$ compared with UT in MOLT-4 T leukemic cells. (C) Jurkat cells were pretreated with $75 \mu \mathrm{M}$ of Ac-DEVD CHO (caspase-3 inhibitor) (Casp-3 Inh) for $2 \mathrm{~h}$ and subsequently treated with cycloleucine $(40$ and $80 \mathrm{mM})$ for $6 \mathrm{~h}$. DNA fragmentation data from three separate experiments, run in duplicate, are normalized to UT values (which are set to 1) and expressed as mean \pm S.E.M. $* P<0.001$ compared with UT values. ${ }^{* *} P<0.001$ compared with baseline values. 
inhibition was also observed in a different leukemic $\mathrm{T}$ cell line (MOLT-4) (Figure 7B).

Furthermore, to evaluate whether apoptotic cell death induced by MAT II inhibition was caspase-3 dependent, the effect of the highly specific reversible inhibitor of caspase-3, DEVD-CHO $(75 \mu \mathrm{M})$, was studied in Jurkat T leukemic cells. The data showed that caspase-3 inhibition effectively blocked apoptotic cell death, indicating that MAT II inhibition leads to caspase-3-dependent apoptotic cell death in T leukemic cells (Figure 7C).

Effect of MAT $2 A$ knockdown on FasL expression and cell viability

To further validate our observations that constitutively expressed MAT II plays a critical role in the survival of T leukemic cells and that its inhibition leads to the induction of FasL expression and apoptotic death, we used siRNA to silence the expression of MAT $2 A$, which encodes the catalytically active subunits of the MAT II isozyme.

A functional MAT $2 A$ siRNA, which consistently gave a knockdown efficiency of $50-70 \%$, was selected from three different 21-mer siRNAs designed based on the human MAT $2 A$ mRNA sequence (GenBank Accession No.
A

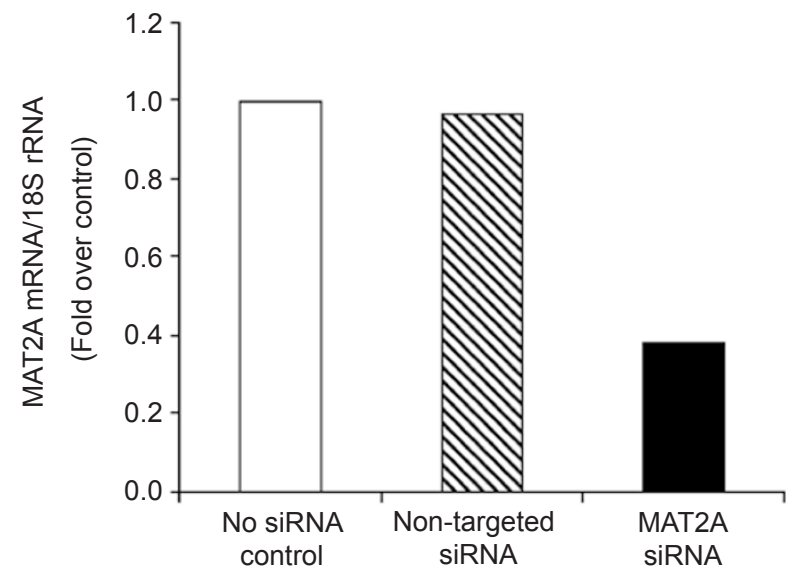

C

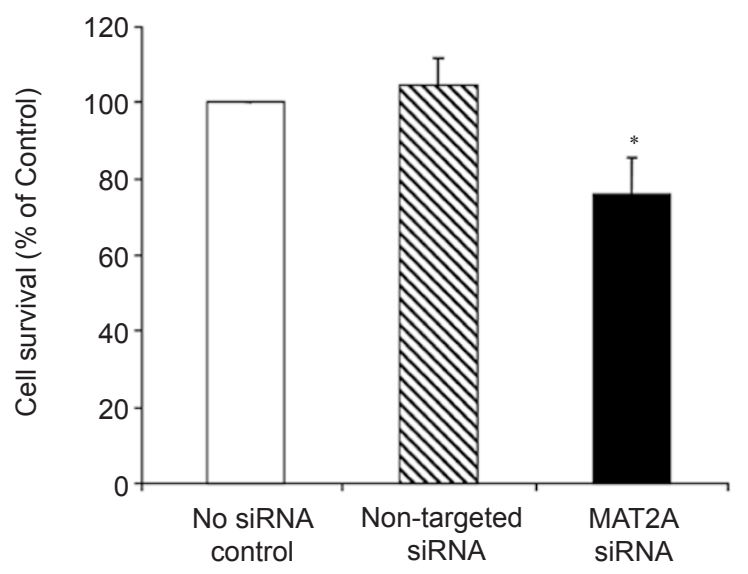

B
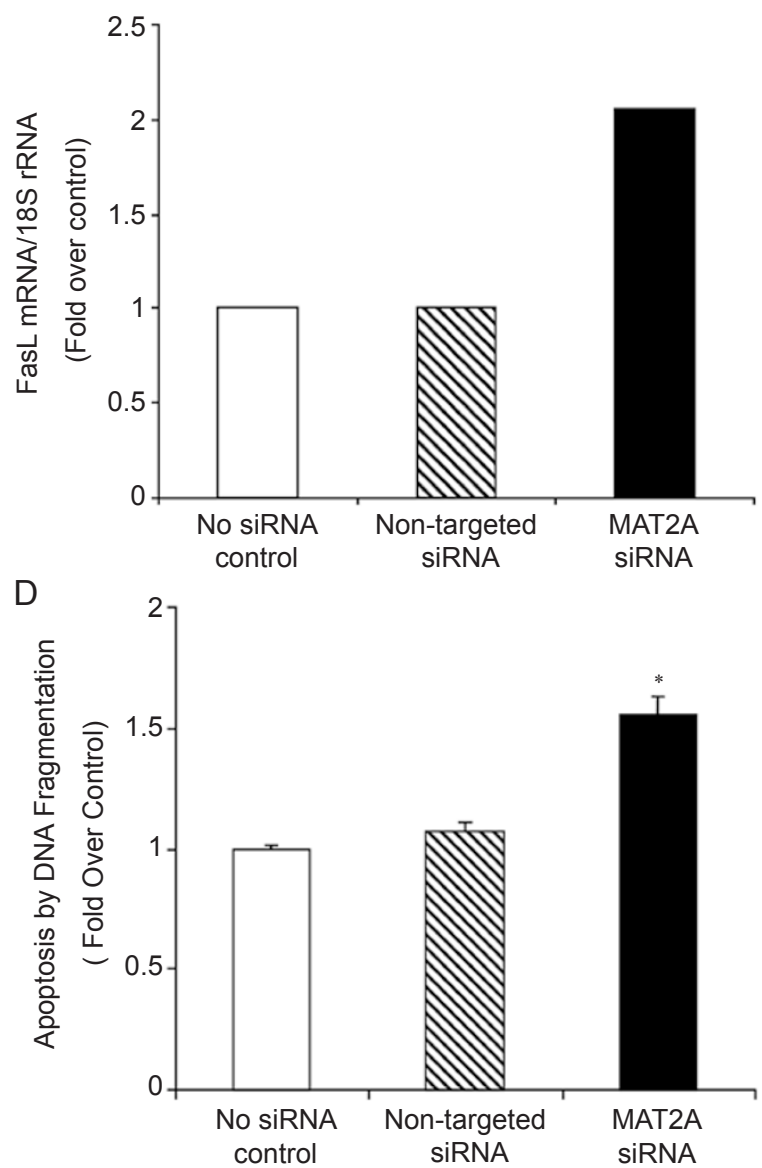

Figure 8 MAT 2A gene knockdown using siRNA results in up-regulation of FasL expression and loss of cell survival. Jurkat T cells were transfected either with MAT 2A siRNA or with non-targeted siRNA. Electroporated cells with only transfection reagent (Nucleofector) served as untreated no-siRNA controls. At $72 \mathrm{~h}$ post transfection, measurements of MAT $2 A$ and FasL mRNA were carried out by real-time PCR and cell survival was measured by Trypan blue dye exclusion assay. (A) MAT 2A siRNA transfection resulted in $\sim 50 \%$ reduction in MAT $2 A$ mRNA expression. (B) MAT $2 A$ gene knockdown led to an up-regulation (two-fold) of Fas $L$ gene expression. (C) Jurkat T cells transfected with MAT 2A siRNA showed a significant decrease in cell survival by $\sim 32 \%$ as compared with the cells transfected with non-targeted siRNA. Data are representative of three separate experiments and are expressed as mean \pm SD. $* P<0.01$ compared with non-targeted siRNA. (D) Transfection of Jurkat $T$ cells with MAT 2A siRNA resulted in apoptotic cell death. Data are representative of three separate experiments and are expressed as mean $\pm \mathrm{SD}$. $* P<0.01$ compared with non-targeted siRNA. 
NM 005911) and used in all the experiments. Cells electroporated with only Nucleofector (transfection reagent) served as an untreated no-siRNA control, and electroporation with non-targeting siRNA served as a negative control in all experiments. Jurkat cells were treated with $3 \mu \mathrm{g} M A T 2 A$ and non-targeting siRNA molecules for $72 \mathrm{~h}$ prior to mRNA and cell viability analyses. MAT $2 A$ mRNA expression in Jurkat T cells was significantly inhibited $(\sim 50 \%)$ by the MAT $2 A$ siRNA (Figure $8 \mathrm{~A}$ ). Knockdown of MAT $2 A$ led to a significant increase of FasL mRNA expression (Figure 8B). These findings indicate that similar to the inhibition of MAT II activity by cycloleucine, specific knockdown of MAT $2 A$ expression leads to the induction of FasL expression and concomitant cell death in Jurkat $\mathrm{T}$ leukemic cells (Figure 8C). Moreover, these MAT 2A siRNA-treated cells showed a significant increase in DNA fragmentation indicative of apoptotic death (Figure 8D).

\section{Discussion}

Seminal studies by Kotb et al. [8-10] have shown that MAT II activity is differentially expressed in normal and leukemic T cells. Normal resting $\mathrm{T}$ lymphocytes have minimal MAT II activity, whereas activated proliferating $\mathrm{T}$ lymphocytes and transformed $\mathrm{T}$ leukemic cells show significantly enhanced MAT II activity [8-10]. Hence, we sought to examine the role of MAT II in the survival of leukemic $\mathrm{T}$ cells. Jurkat cells are continuously proliferating cells derived from human acute lymphoblastic leukemia, and they represent a relevant model to study the molecular mechanisms involved in T cell leukemias [26, 27]. Additionally, to eliminate the possibility of cell linespecific effects, the effect of MAT II inhibition was also examined in another leukemic T cell line, MOLT-4.

Studies have clearly shown that the Fas/FasL pathway is a key regulator of apoptotic $\mathrm{T}$ cell death and is also involved in drug-induced apoptosis of leukemic cells [28]. To investigate the significance of MAT II activity and SAMe biosynthesis in the survival of T leukemic cells, we examined the effect of MAT II inhibition on the Fas/ FasL pathway in Jurkat T leukemic cells. FasL expression plays a major role in the apoptotic cell death of mature $\mathrm{T}$ cells and $\mathrm{T}$ cell hybridomas $[14,29]$. The crucial role of FasL in the regulation of $\mathrm{T}$ cell survival strongly indicates that its expression must be tightly regulated. We showed that MAT II inhibition led to an up-regulation of FasL expression (at both mRNA and protein levels) in these cells. Importantly, apoptotic cell death induced by MAT II inhibition was significantly attenuated by the Fas neutralizing antibody NOK-2, demonstrating the functional relevance of FasL in these cells. Although neu- tralization of FasL significantly protected Jurkat $\mathrm{T}$ cells from apoptotic cell death, the NOK-2 antibody could not completely abrogate DNA fragmentation, suggesting the possible involvement of both Fas-dependent and Fasindependent pathways.

Activation of Fas by FasL leads to its trimerization and subsequent recruitment of the adaptor molecule FADD and procaspase-8, forming the DISC [15-17]. DISC formation and the level of FLICE recruitment to the DISC are known to regulate the Fas-DISC-associated apoptotic cell death in T cells [30]. Our data show that in $\mathrm{T}$ leukemic cells, commensurate with the level of MAT II inhibition, cellular SAMe levels decreased, whereas FasL mRNA and protein expression as well as the DISC assembly involving the recruitment of FADD and procaspase- 8 increased. Consequently, inhibition of MAT II led to enhanced processing and activation of pro-caspase- 8 , which was reflected in the resultant caspase- 8 activity. Importantly, our data showed that caspase- 8 is the critical initiator of apoptosis in T leukemic cells in response to MAT II inhibition. Additionally, as has been reported for type II cells, the Fas-mediated apoptotic pathway induced in response to MAT II inhibition was observed to be linked to the mitochondrial pathway.

FasL-mediated apoptotic cell death in T cells is mainly regulated by c-FLIP. There are two isoforms of cFLIP, c-FLIP S $_{\mathrm{S}}$ and c-FLIP , both of which can be recruited to liganded Fas $[20,21]$. Although the role of c-FLIP $P_{L}$ is controversial, c-FLIPs has been shown to be antiapoptotic and confers resistance to Fas-mediated apoptosis by blocking the proteolytic activation of caspase- 8 in $\mathrm{T}$ lymphocytes [18-23]. Indeed, studies have shown that c-FLIP s plays a major anti-apoptotic role and prevents Fas-mediated apoptotic death in adult $\mathrm{T}$ leukemia and lymphoma cells [18, 23, 31-34]. Our data showed that MAT II inhibition down-regulates c-FLIPs expression in a dose-dependent manner and reinstates Fas-mediated apoptotic signaling and cell death in T leukemic cells.

Induction of the Fas-mediated apoptotic death in response to MAT II inhibition suggests that the transmethylation pathway regulated by MAT II activity and SAMe biosynthesis plays a critical role in the survival of T leukemic cells. It is possible that DNA methylation events may play a role in suppressing Fas-mediated apoptotic signaling in T leukemic cells. Indeed, DNA hypermethylation has been shown to play a causal role in oncogenesis via the inactivation of the transcription of tumor suppressor genes $[35,36]$. Recent reports have shown that transcriptional induction of two critical components of the Fas signaling pathway, namely FasL and caspase- 8 , can be silenced by DNA hypermethylation in 
T leukemic cells $[37,38]$. Thus, it is reasonable to hypothesize that reduced levels of the critical methyl donor, SAMe, caused by MAT II inhibition could affect DNA methylation and potentially restore the transcriptional activation of FasL and caspase-8 leading to apoptotic death. This potential epigenetic control of FasL and caspase- 8 expression is currently being investigated.

Overall, our study indicates that MAT II activity and possibly SAMe-dependent transmethylation can regulate Fas-mediated apoptotic pathways and play a critical role in the survival of human T leukemic cells. Moreover, as MAT II activity is known to be expressed at significantly higher levels in transformed but not in normal quiescent $\mathrm{T}$ lymphocytes, our results also suggest that it could be a potential therapeutic target for the induction of Fasmediated apoptotic death in T leukemic cells.

\section{Materials and Methods}

\section{Cell culture and treatment}

Jurkat (clone E6-1), I 9.2 (caspase-8 mutant of the wild-type Jurkat cell line, clone A3) and MOLT-4 T cells were obtained from ATCC (ATCC, Rockville, MD) and were grown in RPMI 1640 that was supplemented with $10 \%$ fetal bovine serum (FBS), 10 $\mathrm{U} / \mathrm{ml}$ of penicillin and $10 \mu \mathrm{g} / \mathrm{ml}$ of streptomycin, and grown in a $37^{\circ} \mathrm{C}$ and $5 \% \mathrm{CO}_{2}$ environment. Prior to treatment, the cells were maintained in $1 \%$ serum RPMI overnight, after which they were transferred to $10 \%$ serum RPMI and were resuspended at a cell density of $1 \times 10^{6}$ cells $/ \mathrm{ml}$.

\section{Reagents}

Cell culture reagents were obtained from Invitrogen (Carlsbad, CA). Trypan blue was obtained from Life Technologies Inc. (Rockville, MD). Cycloleucine (1-amino-1-cyclopentanecarboxylic acid) and PHA were purchased from Sigma (St Louis, MO). Caspase-3 inhibitor (Ac-DEVD-CHO) was purchased from BIOMOL Research Labs, Inc. (Plymouth, MI). Anti-human FasL, NOK-2 antibody and the isotype $\mathrm{IgG}_{2 \mathrm{a}}$ were purchased from BD Biosciences Pharmingen (San Diego, CA). Caspase-8 inhibitor II and anti-FLIP $\gamma / \delta$ (191-209) antibody were purchased from Calbiochem (La Jolla, CA). Fas-L (C-178), FAS (B-10), caspase- 3 and $\beta$-actin antibodies were purchased from Santa Cruz Biotechnology (Santa Cruz, CA). Caspase-8 (Ab-3) antibody was purchased from Oncogene (La Jolla, CA) and FADD antibody was purchased from Cell Signaling Technology (Beverly, MA).

\section{MAT II activity and S-adenosylmethionine (SAMe) levels}

MAT II activity was assayed in extracts prepared from cell pellets by three cycles of freeze-thawing, as per the procedure described previously [9], with minor modifications. MAT activity was assayed by using $20 \mu \mathrm{M}$ L-Met, $5 \mathrm{mM}$ ATP in $50 \mathrm{mM}$ TES buffer, pH 7.4, $50 \mathrm{mM} \mathrm{KCl}, 15 \mathrm{mM} \mathrm{MgCl}_{2}, 0.3 \mathrm{mM}$ EDTA and $4 \mathrm{mM}$ DTT. One unit of MAT activity was defined as the amount of enzyme that catalyzes the formation of $1 \mathrm{nmol}$ of SAMe in $1 \mathrm{~h}$. SAMe catalyzed by MAT II as well as intracellular SAMe levels were assayed by reverse-phase HPLC from deproteinized extracts prepared by using $4 \%$ metaphosphoric acid (MPA) as described previously [39]

\section{Lactate dehydrogenous assay (LDH assay)}

Lactate dehydrogenase (LDH) activity was measured at 340 nm, using the LDH enzyme assay kit purchased from Sigma Chemical Co. (St Louis, MO). The data were normalized to UT values, which were set to 1 .

\section{Trypan Blue dye exclusion}

To measure viability, cells were stained with Trypan Blue dye and then counted by a method described elsewhere [40]. Cell survival was quantified by measuring the number of dye-excluding cells and the values were normalized to UT values, which were set to $100 \%$.

\section{DNA fragmentation ELISA assay}

Treated cells were lysed after 6-8 h to measure apoptosis. DNA fragmentation was quantitated by determination of monoand oligonucleosomes present in the cytoplasmic fraction of cell lysates using the cell death ELISA kit (Roche, Indianapolis, IN).

\section{Caspase-activity assay}

Cytoplasmic extracts from treated cells were analyzed for caspase-3 activity using the caspase-3 fluorometric assay kit (R\&D Systems, Inc., Minneapolis, MN), and caspase- 8 activity using the CASPASE-8 colorimetric activity assay kit (Chemicon International, Temecula, CA) as per the manufacturer's instructions. The data were normalized to UT values, which were set to 1 .

\section{Immunoprecipitation (IP) and immunoblot analysis}

IP and immunoblot analysis of the CD95 DISC components, including Fas, Fas L, FADD, FLIP $\gamma / \delta$, caspase- 3 and -8 were performed according to the procedure described earlier [41].

\section{RNA isolation, RT-PCR and real-time PCR analysis}

RT-PCR assays were used to assess FasL and MAT $2 A$ mRNA levels in Jurkat cells. Total RNA was isolated from treated cells after 3 and 6 h, using TRIZOL (Invitrogen, Carlsbad, CA). FasL and MAT $2 A$ mRNA levels were assessed by RT-PCR and real-time PCR as described earlier [41]. The specific primers were designed for human GAPDH, FasL and MAT 2A using Primer3 software program; 18S rRNA primer was purchased from SuperArray Bioscience Corporation (Frederick, MD, USA). The following primers were used in real-time PCR:

hGAPDH-FP: 5' TGG GCT ACA CTG AGC ACC AG 3'

hGAPDH-RP: 5' GGG TGT CGC TGT TGA AGT CA 3'

hFasL-FP: 5' GGC CTG TGT CTC CTT GTG AT 3'

hFasL-RP: 5'TGC CAG CTC CTT CTG TAG GT 3'

hMAT IIA-FP: 5'ACA ATC TAC CAC CTA CAG CCA AGT 3'

hMAT IIA-RP: 5' GCA TAA GAG ACC TGA ACA AGA ACC 3'

\section{FasL neutralization assay}

FasL-dependent cell death was evaluated by a neutralization assay. Briefly, Jurkat cells were treated with cycloleucine and 1 $\mathrm{h}$ after treatment the NOK-2 antibody (NOK-2) or the isotype control (IgG) was added to the cells and then harvested after $6 \mathrm{~h}$ for DNA fragmentation measurement using the cell death ELISA kit (Roche, Indianapolis, IN). 


\section{Assessment of mitochondrial transmembrane potential $\left(\Delta \Psi_{m}\right)$}

In order to monitor changes in the mitochondrial membrane potential $\left(\Delta \Psi_{\mathrm{m}}\right), 1 \times 10^{6}$ Jurkat $\mathrm{T}$ cells were treated with increasing concentrations of cycloleucine $(40 \mathrm{mM})$ for $6 \mathrm{~h}$. Changes in mitochondrial $\Delta \Psi_{\mathrm{m}}$ were analyzed as described earlier [41].

\section{MAT2A siRNA transfection}

Three different 21-mer Silencer ${ }^{\circledR}$ pre-designed siRNAs for MAT 2A (GenBank Accession No. NM_005911) and nontargeting siRNA (negative control) were purchased from Ambion (Ambion, Inc., Austin, TX, USA). After a comparative analysis, siRNA with 5'-3' (sense) GGG UGA UGC UGG UUU GAC Utt and (anti-sense) AGU CAA ACC AGC AUC ACC Ctg, which consistently achieved a 50-70\% MAT2A inhibition, was used in all the experiments. Transient transfection was achieved by electroporation with $3 \mu \mathrm{g}$ of MAT $2 \mathrm{~A}$ siRNA per $5 \times 10^{6}$ cells, using the Cell Line Nucleofector Kit V for Jurkat Cells (Amaxa Inc., Gaithersburg, MD, USA). Cells were also electroporated using only the transfection reagent, Nucleofector (no siRNA control), and $3 \mu \mathrm{g}$ of non-targeting siRNA as negative controls.

\section{Statistical analyses}

All data are expressed as mean \pm S.E.M. The data were analyzed by one-way analysis of variance (ANOVA), with the Tukey-Kramer multiple-comparison test for the determination of statistical significance ( $P$ value) on the effect of MAT II inhibition and subsequent SAMe depletion on T leukemic cells. Differences were considered statistically significant for $P<0.05$.

\section{Acknowledgments}

This work was supported by National Institutes of Health grants AA014371 (Barve) and AA015970 (McClain), and the Office of Dietary Supplements, NIH, USA.

\section{References}

1 Cantoni GL. S-adenosylmethionine: a new intermediate formed enzymatically from L-methionine and adenosine triphosphate. J Biol Chem 1953; 204:403-416.

2 Mato JM, Alvarez L, Ortiz P, Pajares MA. S-adenosylmethionine synthesis: molecular mechanisms and clinical implications. Pharmacol Ther 1997; 73:265-280.

3 Bottiglieri T. S-adenosyl-L-methionine (SAMe): from the bench to the bedside-molecular basis of a pleiotropic molecule. Am J Clin Nutr 2002; 76 Suppl:1151S-1157S.

4 Kotb M, Geller AM. Methionine adenosyltransferase: structure and function. Pharmacol Ther 1993; 59:125-143.

5 De La Rosa J, Ostrowski J, Hryniewicz MM, et al. Chromosomal localization and catalytic properties of the recombinant $\alpha$ subunit of human lymphocyte methionine adenosyltransferase. J Biol Chem 1995; 270:21860-21868.

6 Halim AB, LeGros L, Chamberlin ME, Geller A, Kotb M. Regulation of the human MAT2A gene encoding the methionine adenosyltransferase, MAT II: gene organization, promoter characterization and identification of a site in the proximal promoter that is essential for its activity. J Biol Chem 2001;
276:9784-9791.

7 LeGros L, Halim AB, Chamberlin ME, Geller A, Kotb M. Regulation of the human MAT2B gene encoding the regulatory $\beta$ subunit of methionine adenosyltransferase, MAT II. $J$ Biol Chem 2001; 276:24918-24924.

8 Kotb M, Kredich NM. S-adenosylmethionine synthetase from human lymphocytes. Purification and characterization. J Biol Chem 1985; 260:3923-3930.

9 De La Rosa J, LeGros Jr HL, Geller AM, Kotb M. Changes in the relative amount of subunits of methionine adenosyltransferase in human lymphocytes upon stimulation with a polyclonal T cell mitogen. J Biol Chem 1992; 267:10699-10704.

10 LeGros Jr HL, Geller AM, Kotb M. Differential regulation of methionine adenosylmethionine transferase in superantigen and mitogen stimulated human T lymphocytes. J Biol Chem 1997; 272:16040-16047.

11 Zeng Z, Yang H, Huang ZZ, Chen C, Wang J, Lu SC. The role of $\mathrm{c}-\mathrm{Myb}$ in the up-regulation of methionine adenosyltransferase 2A expression in activated Jurkat cells. Biochem J 2001; 353 Pt 1:163-168.

12 Sufrin JR, Coulter AW, Talalay P. Structural and conformational analogues of L-methionine as inhibitors of the enzymatic synthesis of S-adenosyl-L-methionine. IV. Further mono-, biand tricyclic amino acids. Mol Pharmacol 1978; 15:661-677.

13 Lombardini JB, Sufrin JR. Chemotherapeutic potential of methionine analogue inhibitors of tumor derived methionine adenosyltransferases. Biochem Pharmacol 1983; 32:489-495.

14 Brunner T, Wasem C, Torgler R, Cima I, Jakob S, Corazza N. Fas (CD95/APO-1) ligand regulation in T cell homeostasis, cell-mediated cytotoxicity and immune pathology. Semin Immunol 2003; 15:167-176.

15 Chinnaiyan AM, Tepper CG, Seldin MF, et al. FADD/MORT1 is a common mediator of CD95 (Fas/APO-1) and tumor necrosis factor receptor-induced apoptosis. J Biol Chem 1996; 271:4961-4965.

16 Medema JP, Scaffidi C, Kischkel FC, et al. FLICE is activated by association with the CD95 death-inducing signaling complex (DISC). EMBO J 1997; 16:2794-2804.

17 Peter ME, Krammer PH. The CD95 (APO-1/Fas) DISC and beyond. Cell Death Differ 2003; 10:26-35.

18 Irmler M, Thome M, Hahne M, et al. Inhibition of death receptor signals by cellular FLIP. Nature 1997; 388:190-195.

19 Sharp DA, Lawrence DA, Ashkenazi A. Selective knockdown of the long variant of cellular FLICE inhibitory protein augments death receptor-mediated caspase- 8 activation and apoptosis. J Biol Chem 2005; 280:19401-19409.

20 Krueger A, Baumann S, Krammer PH, Kirchoff S. FLICEinhibitory proteins: regulators of death receptor-mediated apoptosis. Mol Cell Biol 2001; 21:8247-8254.

21 Thome M, Tschopp J. Regulation of lymphocyte proliferation and death by FLIP. Nat Rev Immunol 2001; 1:50-58.

22 Rasper DM, Vaillancourt JP, Hadano S, et al. Cell death attenuation by 'Usurpin', a mammalian DED-caspase homologue that precludes caspase- 8 recruitment and activation by the CD95 (Fas, APO-1) receptor complex. Cell Death Differ 1998; 5:271-288.

23 Schmitz I, Weyd H, Krueger A, et al. Resistance of short term activated $\mathrm{T}$ cells to CD95-mediated apoptosis correlates with de novo protein synthesis of c-FLIP(short). J Immunol 2004; 
172:2194-2200.

24 Scaffidi C, Fulda S, Srinivasan A, et al. Two CD95 (APO-1/ Fas) signaling pathways. EMBO J 1998; 17:1675-1687.

$25 \mathrm{Li} \mathrm{H}$, Zhu X, Xu C, Yuan J. Cleavage of BID by caspase- 8 mediates the mitochondrial damage in the Fas pathway of apoptosis. Cell 1998; 94:491-501.

26 Bremer E, Samplonius DF, Peipp M, et al. Target cell-restricted apoptosis induction of acute leukemic T cells by a recombinant tumor necrosis factor-related apoptosis-inducing ligand fusion protein with specificity for human CD7. Cancer Res 2005; 65:3380-3388.

27 Jung KC, Kim NH, Park WS, Park SH, Bae Y. The CD99 signal enhances Fas-mediated apoptosis in the human leukemic cell line, Jurkat. FEBS Lett 2003; 554:478-484.

28 Friesen C, Herr I, Krammer PH, Debatin KM. Involvement of the CD95 (APO-1/Fas) receptor/ligand system in drug induced apoptosis in leukemic cells. Nat Med 1996; 2:574-577.

29 Griffith TS, Brunner T, Fletcher SM, Green DR, Ferguson TA. Fas ligand-induced apoptosis as a mechanism of immune privilege. Science 1995; 270:1189-1192.

30 Peter ME, Kischkel FC, Scheuerpflug CG, Medema JP, Debatin KM, Krammer PH. Resistance of cultured peripheral T cells towards activation-induced cell death involves a lack of recruitment of FLICE (MACH/caspase-8) to the CD95 death-inducing signaling complex. Eur J Immunol 1997; 27:1207-1212.

31 Mathias S, Leitz A, Anagnostopoulos I, et al. c-FLIP mediates resistance of Hodgkin/Reed-Sternberg cells to death receptorinduced apoptosis. J Exp Med 2004; 199:1041-1052.

32 Dutton A, O'Neil JD, Milner AE, et al. Expression of the cellular FLICE-inhibitory protein (c-FLIP) protects Hodgkin's lymphoma cells from autonomous Fas-mediated death. Proc Natl Acad Sci USA 2004; 101:6611-6616.

33 Krueger A, Fas SC, Giaisi M, et al. HTLV-1 tax protects against CD95-mediated apoptosis by induction of the cellular
FLICE-inhibitory protein (c-FLIP). Blood 2006; 107:39333939.

34 Okamoto K, Fujisawa J, Reth M, Yonehara S. Human T-cell leukemia virus type-I oncoprotein Tax inhibits Fas-mediated apoptosis by inducing cellular FLIP through activation of NFkappaB. Genes Cells 2006; 11:177-191.

35 Esteller M, Corn PG, Baylin SB, Hemran JG. A gene hypermethylation profile of human cancer. Cancer Res 2001; 61:32253229.

36 Jones PA, Takai D. The role of DNA methylation in mammalian epigenetics. Science 2001; 293:1068-1070.

37 Yasunaga J, Taniguchi Y, Nosak K, et al. Identification of aberrantly methylated genes in association with adult T-cell leukemia. Cancer Res 2004; 64:6002-6009.

38 Hopkins-Donaldson S, Zielger A, Kurtz S, et al. Silencing of death receptor and caspase- 8 expression in small cell lung carcinoma cell lines and tumors by DNA methylation. Cell Death Differ 2003; 10:356-364.

39 Song Z, Chen T, Deaciuc IV, et al. Modulation of endotoxin stimulated interleukin-6 production in monocytes and kupffer cells by S-adenosylmethionine (SAMe). Cytokine 2004; 28:214-223.

40 McClowskey TW, Chavan S, Lakshmi Tamma SM, Pahwa S. Comparison of seven quantitative assays to assess lymphocytic cell death during HIV infection: measurement of induced apoptosis in anti-Fas treated Jurkat cells and spontaneous apoptosis in peripheral blood mononuclear cells from children infected with HIV. AIDS Res Hum Retroviruses 1998; 14:1413-1422.

41 Uriarte S, Joshi-Barve S, Song Z, et al. Akt inhibition upregulates FasL, downregulates c-FLIP s $_{\mathrm{s}}$ and induces caspase8 -dependent cell death in Jurkat T lymphocytes. Cell Death Differ 2005; 12:233-242. 